\title{
Sistemas de estacionamento vertical modulado em estrutura metálica
}

\author{
Ezequiel Mendonça Rezende \\ Mestre em Construções Metálicas pela Universidade Federal de Ouro Preto, Especialista em Arquitetura em \\ Construções Metálicas e Arquiteto Urbanista formado pelo Centro Universitário Izabela Hendrix \\ E-mail:emrezende@gmail.com

\begin{abstract}
Antônio Maria Claret de Gouveia
Professor, Universidade Federal de Ouro Preto - Escola de Minas - Departamento de Engenharia Civil Programa de Pós-Graduação em Engenharia Civil - Mestrado em Construções Metálicas

E-mail:amclaret@pesquisador.cnpq.br
\end{abstract}

\section{Resumo}

Um dos maiores problemas das grandes cidades está relacionado ao envelhecimento dos centros urbanos associado à falência de toda a estrutura viária para receber um sistema de trânsito cada vez mais conturbado. Em função de toda essa situação, vêm sendo implementadas políticas públicas no sentido de revitalizar os centros urbanos. Concomitantemente, vem ocorrendo uma procura de melhoria nos transportes coletivos. Assim, espera-se resolver parte desse quadro de problemas, o qual vem sendo intensificado com a questão da falta de vagas para o estacionamente de veículos nos referidos centros urbanos. Surge, dessa forma, um novo enfoque para a questão dos estacionamentos, sendo, então, criadas novas propostas para a solução de tal problema.

Para a criação de novos espaços de estacionamento nessas regiões centrais, é necessário buscar soluções criativas que adotem um aumento da densidade de armazenamento de veículos em determinados locais. Uma das possibilidades é a implantação de edifícios modulados em estrutura metálica, totalmente automatizados, que consistem, praticamente, em simples estruturas, sem a necessidade do uso de lajes para pisos, ou mesmo, de elementos de fechamentos. Como exemplo desses edifícios modulados, podemos citar os galpões de armazenamento de grandes atacadistas existentes no Brasil. Esses edifícios, por suas próprias características, não permitem o acesso do usuário ao seu interior, já que os veículos são conduzidos por equipamentos totalmente robotizados.

Palavras-chave: Estrutura metálica, garagem, estacionamento, páletes, sistemas industrializados, sistemas modulados, sistema de estacionamento modular automatizado, MAPS, reestruturação, automação, veículo, carro, automóvel, congestionamento urbano, pedágio urbano.

\begin{abstract}
One of the greatest problems of a big city is associated with the aging of urban downtown centers and the failure of the traffic structure to support a traffic system that becomes more and more chaotic every day. While a growth of public policies which encourage downtown revitalization and the improvement of mass transportation should help revert part of this scenario, ironically it aggravates the already complex parking problem in urban downtown centers. This issue requires a new approach as well as a search for new solutions.

It is imperative to look for creative solutions when planning new parking locations in downtown areas by adopting denser vehicle storage in specific sites. One possibility is the implementation of buildings with modular metallic structures, fully automated, comprised of a simple structure without the need for floor and wall fillings. Take for example the large warehouses in Brazil; these buildings, because of their intrinsic characteristics, do not allow the user access to its interior as the vehicles are conducted by fully robotized equipment.
\end{abstract}

Keywords: Metallic structure, garage, parking, pallets, industrialized systems, modulated systems, modular automated parking systems, MAPS, retrofit, automation, vehicle, car, automobile, urban congestion, congestion charge. 


\section{Introdução}

O envelhecimento dos centros urbanos, associados à falência da estrutura viária, tem apresentado grandes problemas para receber um sistema de trânsito cada vez mais conturbado. A melhoria dos transportes coletivos e a implantação de políticas pública para a revitalização dos centros urbanos poderão reverter parte desse quadro, mas, em contrapartida, agrava-se o complexo problema de estacionamento de veículos nestas áreas centrais. Um novo enfoque sobre essa questão e buscas de novas soluções para o problema de estacionamento se constituem no tema principal desse trabalho.

Para compreender melhor o impacto urbano provocado pelo uso do automóvel, deve-se retroceder a sua evolução na história, desde a sua criação, no final do séc. XIX, até o seu grande crescimento após a segunda guerra mundial. Atualmente foram criadas nos grandes centros urbanos, políticas de pedágios urbanos com o objetivo de minimizar os impactos negativos do trânsito em determinadas áreas.

É necessário buscar soluções criativas, que privilegiam o aumento da densidade de armazenamento de veículos em determinados locais da cidade. A implantação de edifícios modulados em estrutura metálica, totalmente automatizados, atendem a essas soluções. Esses edifícios se constituem, praticamente, em simples estruturas sem a necessidade do uso de lajes para pisos ou mesmo de elementos de fechamento como alvenarias.

\section{Desenvolvimento dos edifícios-garagens automatizados}

Esse trabalho limitou-se ao desenvolvimento de quatro módulos, que representam algumas das principais soluções em Sistema de Estacionamento Modular Automatizado (Modular Automated Parking Systems - MAPS).

Esses módulos apresentam dimensões que podem acomodar a maioria dos tipos de automóveis existentes no Brasil.
As quatro tipologias de Edifícios MAPS apresentados nesse artigo podem ser implantados em uma grande variedade de situações, desde pequenos lotes urbanos até a composições que permitam a criação de edifícios garagens que comportam centenas ou até milhares de veículos, bastando, para isto, agregar mais módulos construtivos à edificação.

Cada módulo é formado por células que comportam dois ou quatro veículos, dependendo da sua tipologia. Por serem em estrutura metálica, esses módulos podem ser montados em um terreno urbano e após um determinado tempo, serem desmontados e transferidos para outro local.

\subsection{Sistema Módulo Lateral - 2}

O desenvolvimento do Sistema Módulo Lateral - 2 partiu de um módulo que comporta dois veículos por pavimento (Figura 1). Poderia utilizar esse tipo de solução em recuos dos edifícios, por exemplo, para o retrofit de dois prédios existentes, utilizando os afastamentos existentes. Deste modo, sem grandes intervenções, seriam criados novos espaços de estacionamentos.

Essa solução esbarra nas imposições legais das legislações de Uso e Ocupação do Solo Urbano de várias cidades, que exigem que as edificações tenham um determinado afastamento. Um questionamento deve ser feito, já que existem situações onde é possível melhorar as disponibilidades de vagas desses antigos edifícios.

Naturalmente essas novas instalações só poderão ser construídas, quando todas as condicionantes de projeto estejam satisfeitas, tais como: a existência de fachadas ou paredes cegas, sem aberturas; possibilidades de acessos no nível da rua ou mesmo a partir do último pavimento de uma garagem existente; e mesmo condições técnicas adequadas para a instalação dos sistemas de garagens.

A tecnologia dos elevadores será semelhante às adotadas nos elevadores convencionas, sendo que, em sua plataforma, deverá existir um dispositivo de transporte horizontal (Dolly), que permita retirar o veículo do compartimento de transferência, colocá-lo sobre a plataforma do elevador e, finalmente, transferi-lo para uma vaga livre.

Em um espaço de apenas 2,50 m x 15,00 m, já seria possível a implantação desse sistema, acomodando dois veículos por pavimento. Em uma situação hipotética, poder-se-ia construir uma estrutura de 10 pavimentos para comportar até 19 veículos, já que se perde uma vaga no nível da rua, para permitir o acesso dos veículos ao elevador. Essa tipologia de MAPS apresenta um rendimento de 12,89 m²/vaga.

\subsection{Sistema Módulo Duplo - 4}

No desenvolvimento do Sistema Módulo Duplo - 4 foi definido que cada módulo poderia comportar dois veículos de cada lado da estrutura, sendo, então, um total de quatro veículos por conjunto de módulo. O espaço entre os módulos seria destinado para o movimento do equipamento transportador, que teriam movimentos horizontais e verticais. Essa solução está representada na Figura 2.

Para capacitar o elevador de movimento horizontal, este deverá ter uma coluna vertical, que se movimenta sobre trilhos dispostos no piso do primeiro pavimento. Guias secundárias dispostas horizontalmente em alguns dos pavimentos acima poderão dar maior estabilidade à estrutura da torre do elevador. Para o movimento vertical do elevador, este utilizará as mesmas tecnologias dos elevadores convencionais. Além da plataforma, o elevador deverá ter um dispositivo de transporte horizontal (Dolly), que fica junto à plataforma do elevador, para permitir a retirada do veículo do compartimento de transferência e colocá-lo sobre a plataforma do elevador e, então, transferi-lo à uma vaga livre.

Para a implantação de cada módulo com quatro veículos por pavimento, é necessário um espaço de 4,50 m x 15,00 m, sendo que uma área livre no centro da estrutura de 4,50 m x 5,00 m deve ser destinada ao ele- 


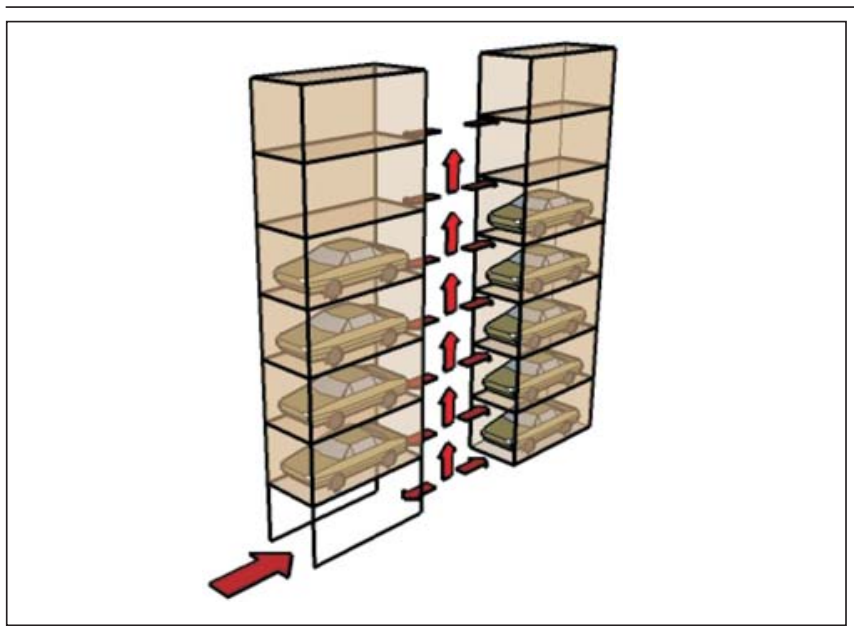

Figura 1 - Sistema Módulo Lateral - 2.

vador. Essa estrutura pode ter uma grande repetição desses módulos, tanto na horizontal, quanto na vertical. O limite na repetição horizontal se dá nas limitações dos terrenos disponíveis. Essa tipologia de MAPS apresenta um rendimento de $11,25 \mathrm{~m}^{2} /$ vaga.

A maior limitação desse sistema será a sua agilidade no tempo de guardar e retirar os veículos, pois um grande número de vagas atendidas por um único dispositivo de transporte fará que o tempo de espera para que o usuário tenha seu veículo devolvido seja até certo limite tolerável. Pelas experiências de sistemas desenvolvidos em outros países, esse limite é de, no máximo, dois minutos. Para reduzir esse tempo, a solução é a utilização de equipamentos de transportes mais velozes ou, então, aumentar o número de equipamentos, o que representa a perda de duas vagas por cada equipamento transportador.

\subsection{Sistema Módulo Circular - 4}

Para o Sistema Módulo Circular - 4 pode-se adotar, como solução, as mesmas configurações do módulo apresentado no item anterior, só que estes serão dispostos em forma radial, totalizando quatro módulos por pavimento, tendo um aspecto final como o representado na Figura 3.

Nesse tipo de sistema, o elevador não tem a capacidade de movimento horizontal, mas apenas movimento vertical e capacidade de rotação da plataforma dos veículos. Esse giro permite que o dispositivo de transferência dos veículos fique em posição adequada em relação à vaga. Esssa tipologia de MAPS apresenta um rendimento de 11,53 m²/vaga.

\subsection{Sistema Módulo Circular - 12}

O Sistema Módulo Circular - 12 apresenta solução semelhante ao sistema anterior, só que a forma radial abriga até doze veículos por pavimento (Figura 4). Para a implantação

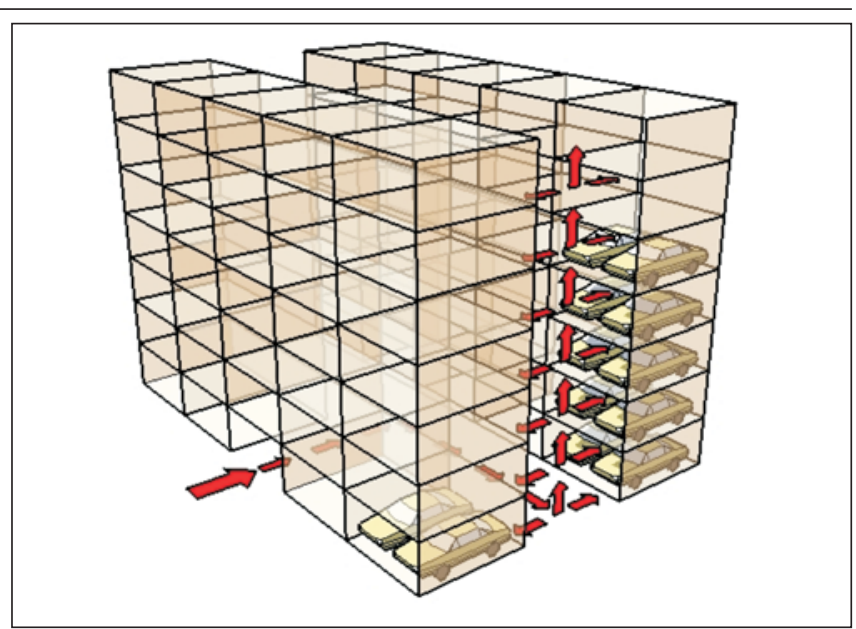

Figura 2 - Sistema Módulo Duplo - 4.

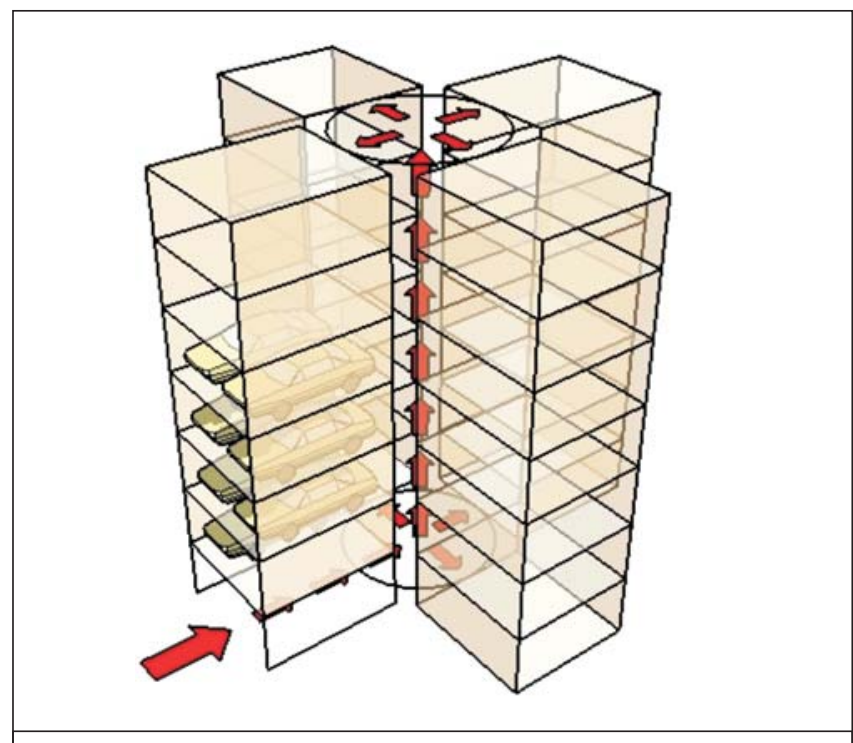

Figura 3 - Sistema Módulo Circular - 4.

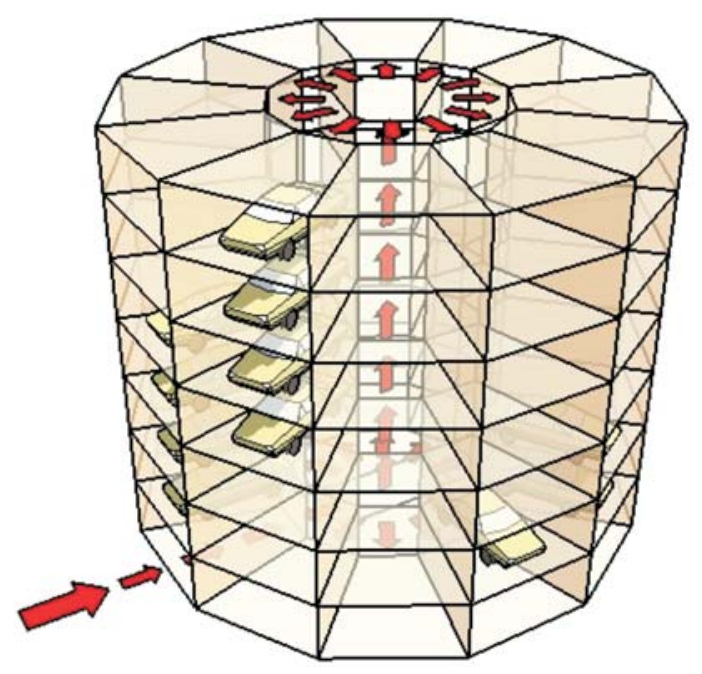

Figura 4 - Sistema Módulo Circular - 12. 
dessa solução, serão necessários espaços muito maiores, mas, em compensação, o número de vagas será muito maior.

A ampliação dessa solução pode ser feita aumentando-se o número de módulos por pavimento e, conseqüentemente, atingindo uma maior otimização dos espaços.

Essa tipologia de MAPS apresenta um rendimento de 17,20 m²/vaga.

\section{Estudo de caso}

Foi lançado, em 1995, um concurso público de projetos que deveria contemplar o espaço urbano da Praça Rui Barbosa em frente do Edifício da Estação Ferroviária, na cidade de Belo Horizonte - MG. Segundo as diretrizes do concurso, deveria ser previsto uma garagem subterrânea para aproximadamente 1000 veículos. Devido a sérias restrições orçamentárias o projeto foi abandonado.

A proposta vencedora era um edifício garagem subterrâneo com sistema estrutural convencional em concreto aramado numa extensão de um pouco mais de 550 m de comprimento, com a capacidade de abrigar 963 veículos e com uma área de aproximadamente $22.900 \mathrm{~m}^{2}$, sendo que destes, $20.184 \mathrm{~m}^{2}$, destinados, exclusivamente, para as vagas e circulação de veículos. Isso representa uma relação de 20,96 m²/vaga.

Comparando esse tipo de edificação com os Sistemas de Estacionamento Modulares Automatizados, pode-se chegar a valores comparativos que evidenciam a vantagem, em determinadas situações, da implantação dos MAPS, tais como menores espaços destinados às vagas, menor consumo de concreto, menores movimentos de terra, menores áreas impermeabilizada, etc.

O estudo de caso apresentado compara a solução de um edifício subterrâneo convencional em concreto armado proposto para essa região com um Edifício MAPS. Para esse estudo, o Edifício MAPS foi considerado, inicialmen- te, acima do solo e, posteriormente, totalmente subterrâneo.

Empregando um Edifício MAPS como o Sistema Módulo Duplo 4, com dois veículos por módulo, 12 módulos da cada lado do edifício e 10 pavimentos, poder-se-ia comportar até 480 veículos. Portanto duas instalações desse tipo ser o suficiente para atender às exigências do edital do concurso, com um total de 960 vagas.

A Tabela 1 apresenta alguns dados que abordam três possibilidade de implantação de garagens: uma convencional subterrânea, Edifício MAPS subterrâneo e, por último, um Edifício MAPS acima do solo.

\subsection{Edifício proposto}

Para a proposta de um Edifício MAPS que atenda a Praça Rui Barbosa, considerou a implantação de dois blocos de edifícios idênticos, acoplados um ao lado do outro, construído todo em

Tabela 1 - Quadro comparativo dos sistemas de garagem.

\begin{tabular}{c|c|c|c}
\hline \multirow{2}{*}{ Tipologia do edifício } & \multirow{2}{*}{$\begin{array}{c}\text { Convencional } \\
\text { subterrâneo }\end{array}$} & \multicolumn{2}{|c}{ Edifício MAPS } \\
\cline { 3 - 4 } & manual & automático & Acima do solo \\
\hline Procedimento de manobra & 1 & 10 & 10 \\
\hline Número de pavimentos & 963 & 96 & 96 \\
\hline Número de vagas/pavimento & 963 & 960 & 960 \\
\hline Número total de vagas & $4 \mathrm{~m}$ & $22 \mathrm{~m}$ & 0 \\
\hline Profundidade do 10 subsolo & $22.867,77 \mathrm{~m}^{2}$ & $1620 \mathrm{~m}^{2}$ & $1620 \mathrm{~m}^{2}$ \\
\hline Área de projeção & $20.184,18 \mathrm{~m}^{2}$ & $10800 \mathrm{~m}^{2}$ & $10800 \mathrm{~m}^{2}$ \\
\hline Área útil para vagas & $22.867,77 \mathrm{~m}^{2}$ & $10800 \mathrm{~m}^{2}$ & $10800 \mathrm{~m}^{2}$ \\
\hline Área total construída & $2,20 \times 4,50 \mathrm{~m}$ & $2,175 \times 5,00 \mathrm{~m}$ & $2,175 \times 5,00 \mathrm{~m}$ \\
\hline Dimensões de cada vaga & $20,96 \mathrm{~m}^{2} / \mathrm{vaga}^{2}$ & $11,27 \mathrm{~m}^{2} / \mathrm{vaga}^{2}$ & $11,27 \mathrm{~m}^{2} / \mathrm{vaga}$ \\
\hline Relação m²/vaga & $51.504,86 \mathrm{~m}^{2}$ & $6936,00 \mathrm{~m}^{2}$ & $1620 \mathrm{~m}^{2}$ \\
\hline Impermeabilização & $118.911 \mathrm{~m}^{3}$ & $1478,40 \mathrm{~m}^{3}$ & 0 \\
\hline Movimento de terra & $15.010,57 \mathrm{~m}^{3}$ & $2288,40 \mathrm{~m}^{3}$ & $486 \mathrm{~m}^{3}$ \\
\hline Consumo de concreto & & &
\end{tabular}


Ezequiel Mendonça Rezende et al.

estrutura metálica e acima do nível do solo. Para uma melhor compreensão, na Figura 5, é apresentado o sistema construtivo proposto com as seguintes observações:

1) Um módulo com capacidade de acomodar até quatro veículos.

2) Disposição dos módulos na horizontal (para o caso desse edifício proposto serão necessários 12 módulos).

3) Disposição dos módulos na vertical (no caso desse edifício proposto serão necessários 10 módulos - ou 20 pavimentos).
4) Bloco de um edifício completo formado por 120 módulos e com capacidade de acomodar até 480 veículos.

5) Dois blocos de edifício idênticos e acoplados (B1 e B2), totalizando 240 módulos com capacidade de acomodar até 960 veículos.

Na Figura 6, é representado o módulo construtivo de dois pavimentos comportando dois veículos por pavimento, totalizando quatro veículos por módulo.

Na Figura 7, está apresentado o detalhe de um bloco da edificação, incluindo parte do sistema de fechamento vertical com comunicação visual, fechamento horizontal, dispositivo transportador (elevador e Dolly), rampa de acesso lateral ou rampa de acesso frontal.

Portanto, para atender ao total de 960 vagas, serão necessários dois Edifícios MAPS que totalizam um consumo de aço de $312.513 \mathrm{~kg}$. Isto equivale a 28,94 $\mathrm{kg} / \mathrm{m}^{2}$, que é um valor razoável para edificações em aço, considerando que essa edificação não necessita de outros componentes estruturais ou mesmo de vedação, exceto os painéis externos em chapas metálicas pintadas, quando necessários.

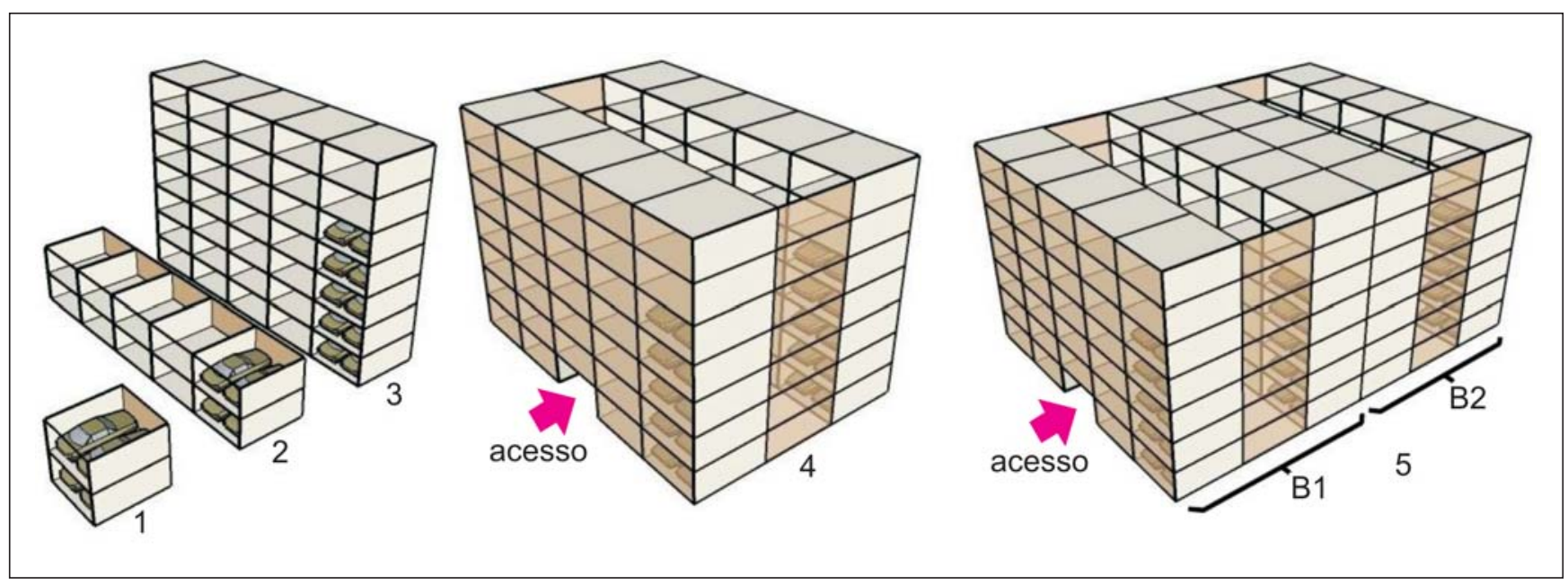

Figura 5 - Diagrama do sistema construtivo modular.

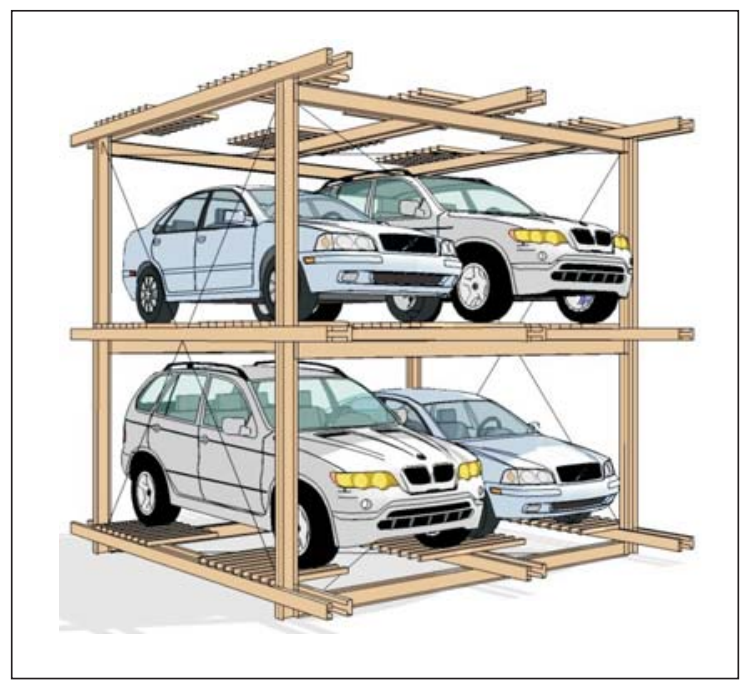

Figura 6 - Módulo construtivo.

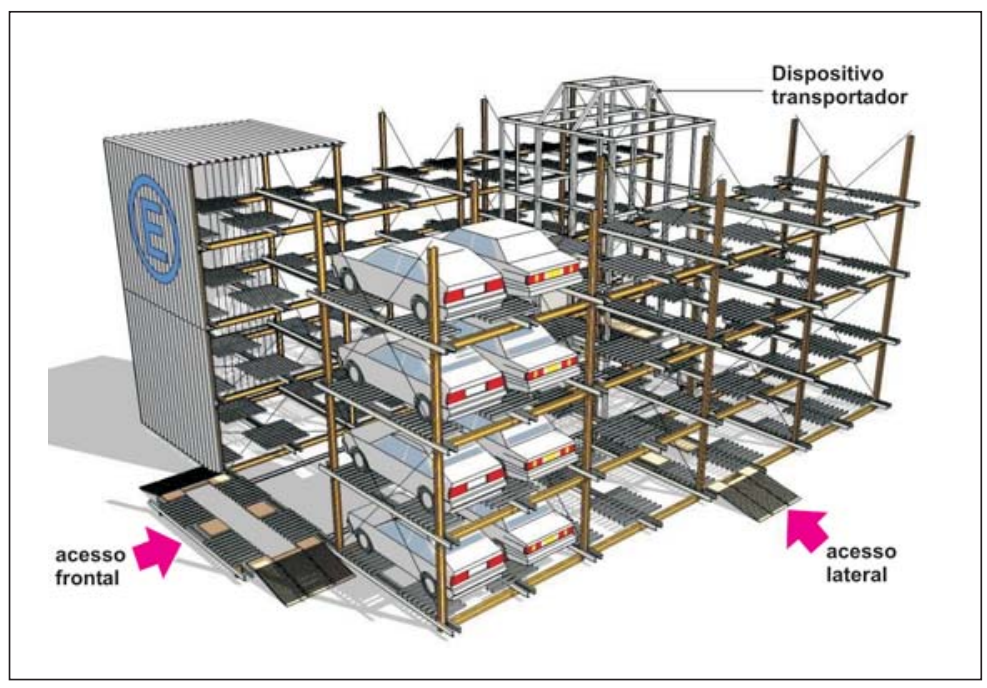

Figura 7 - Estacionamento Modular Automatizado para a Praça Rui Barbosa. 


\subsection{Implantação de garagens em lotes urbanos}

A possibilidade de implantação de edifícios MAPS em uma área urbana depende, exclusivamente, da disponibilidade de terrenos com dimensões mínimas que atendam às exigências do projeto arquitetônico.

Para essa pesquisa, foi escolhida, na região da Savassi em Belo Horizonte MG, uma das principais regiões de comércio de rua da cidade, uma área de 64 quarteirões.

Foi feito o levantamento dos terrenos dessa região e constatou que, dos poucos terrenos ainda vagos, a grande maioria são, relativamente, pequenos, com 10, 12 ou 15 metros de frente por 20 ou 30 metros de profundidade.

Existia uma proposta urbanística para essa região, que, também, não foi executada, chamada de "Street Shopping Savassi”, que previa uma garagem que abrigaria aproximadamente 4000 veículos, distribuídos em três subsolos, totalizando $88.565 \mathrm{~m}^{2}$.
Para atender às exigências desse projeto, seriam necessários dez Edifícios MPAS, que poderiam ser implantados em terrenos com, aproximadamente, $900 \mathrm{~m}^{2}$. Tais edifícios poderiam ter oito pavimentos, abrigando um total de 104 módulos, o que corresponde à 416 vagas.

Por ser praticamente impossível encontrar tais lotes contíguos nessa região ou que tenham exatos $900 \mathrm{~m}^{2}$ de área, e pelas próprias características dos MAPS, estes poderão ser implantados nos lotes ainda disponíveis, seguindo critérios que permitam ter a melhor adaptação e o melhor rendimento em cada terreno, variando o número de módulos e de pavimentos para cada edifício proposto.

\section{Conclusões}

Várias vantagens no uso dessa tecnologia podem ser apresentadas, tais como redução do espaço ocupado pelos veículos, redução no pé-direito das garagens, otimização de espaços, duplicação do número de vagas, não havendo, ainda, a necessidade de elevadores, rampas ou escadas, e, também, não sendo necessário condicionamento de ar ou mesmo ventilação.

Por se tratar de um sistema, onde a modulação é fundamental para o seu desenvolvimento, e por esse tipo de edificação ser totalmente industrializado, é grande a racionalização dos perfis de estrutura metálica.

\section{Referências Bibliográficas}

BRIERLEY, John. Parking of Motor Vehicles. London: C.R. Books Limited, 1962.

KLOSE, Dietrich. Parkhäuser und Tiefgaragen. Stuttgart: Verlag Gerd Hatje, 1965.

NPA Parking Consultants Council. Guide to the Design \& Operation of Automated Parking Facilities - USA, 2003.

RAMALHO, Luiz Carlos de Aquino. Paletização de Estacionamentos. Brasil, 1980.

\section{Artigo recebido em 31/05/2005 e} aprovado em 13/07/2006.

\section{REM - Revista Escola de Minas 70 anos divulgando CIÊNCIA.}

$\star * * * * *$

www.rem.com.br

$\star * * * * *$ 